\title{
p53 Alteration and Chromosomal Instability in Prostatic High-Grade Intraepithelial Neoplasia and Concurrent Carcinoma: Analysis by Immunohistochemistry, Interphase In Situ Hybridization, and Sequencing of Laser-Captured Microdissected Specimens
}

\author{
Jaudah Al-Maghrabi, M.D., FRCPC, Lada Vorobyova, M.D., William Chapman, M.D., FRCPC, \\ Michael Jewett, M.D., FRCS, Maria Zielenska, Ph.D., Jeremy A. Squire, Ph.D. \\ Ontario Cancer Institute (JA-M, LV, WC, MJ, JAS), Princess Margaret Hospital (JA-M, LV, WC, MJ, JAS), \\ Toronto General Hospital (JA-M, WC, MJ, JAS), University Health Network (JA-M, LV, WC, MJ, JAS), and \\ Departments of Laboratory Medicine and Pathobiology (JA-M, LV, SC, JAS), Medical Biophysics (JAS), \\ Surgical Oncology (MJ), and Pediatric Laboratory Medicine, Hospital for Sick Children (MZ), Faculty of \\ Medicine, University of Toronto, Toronto, Ontario, Canada
}

p53 mutation has been shown to be associated with chromosomal instability (CI) in many human dysplastic and neoplastic lesions. However, the precise role of $\mathrm{p53}$ in the pathogenesis of prostate carcinoma (Pca) is unknown. Topographic analysis of p53 alteration using immunohistochemistry (IHC) was performed on 35 archived prostatectomy specimens containing Pca foci; high-grade prostrate intraepithelial neoplasia (HPIN) foci intermingled with cancer (HPINI) and situated away (HPINA). Specimens from 2 patients were topographically genotyped using laser capture microdissection, PCR amplification, and direct sequencing of p53 exons 5-9. CI was evaluated in the same tissue foci by interphase in situ hybridization (IFISH) using centromere probes for chromosomes 7, 8, and Y. p53 immunoreactivity was found in $20 \%, 17 \%, 0$, and 0 in Pca, HPINI, HPINA, and benign epithelium, respectively. p53 molecular analysis in the specimens examined confirmed the IHC findings. IFISH revealed numerical chromosomal alterations in keeping with $\mathrm{CI}$ in $71 \%$ and $25 \%$ of p53+ and p53- Pca, respectively $(P=.1), 67 \%$ and 0 of p53+ and p53HPIN, respectively $(P<.02)$, and in $27 \%$ and 0 of HPINI and HPINA, respectively. We concluded that

Copyright (C) 2001 by The United States and Canadian Academy of Pathology, Inc.

VOL. 14, NO. 12, P. 1252, 2001 Printed in the U.S.A.

Date of acceptance: August 27, 2001

This project was funded by the U.S. Army Medical Research and Materie Command (USAMRMC) Prostate Cancer Research Program (PCRP). Visible Genetics Inc. provided the reagents for p53 sequencing.

Address reprint requests to: Jeremy A. Squire, Ph.D., Division of Cellular and Molecular Biology, Ontario Cancer Institute, Princess Margaret Hospital, 610 University Avenue, Room 9-721, Toronto, Ontario M5G 2M9 Canada; e-mail: jeremy.squire@utoronto.ca; fax: 416-920-5413. p53 mutation is an early change in at least a subset of Pca. HPINI foci tend to have higher overall p53 immunoreactivity and CI than HPINA. The presence of p53 mutation in HPIN was associated with the presence of $\mathrm{CI}$ as determined by IFISH. Our study also provided additional evidence in support of the concept that HPIN might be the earliest precursor of cancer. Furthermore, our studies identify genomic similarities in HPINI and Pca, implying that carcinoma may arise from progression of certain HPIN foci that most likely harbor p53 mutation and/or more $\mathrm{CI}$.

KEY WORDS: Chromosomal instability, Immunohistochemistry, In situ hybridization, p53 sequencing, Prostate carcinoma, Prostate intraepithelial neoplasia.

Mod Pathol 2001;14(12):1252-1262

The reported frequency of mutation of the p53 tumor suppressor gene in Pca has varied widely, ranging from $3-72 \%$ in carcinomas of the prostate and $0-68 \%$ in HPIN $(1-8)$. In the literature, there is controversy about the question of whether p53 alteration is an early or late genetic change $(1,6,7$, $9-15)$. Striking heterogeneity of p53 mutation in prostate cancer has been reported (16), and different mutated alleles were found among multiple tumor foci in single glands $(16,17)$. p53 has been found to be associated with genomic instability leading to chromosomal rearrangement, which in turn has been demonstrated to be a feature of many neoplastic and preneoplastic (dysplastic) human epithelia (18-30). The transition from preinvasive disease to invasive carcinoma was shown to be 
associated with changes in the number of chromosome copy and that coincide with the loss of TP53 function. Whether there is a role of chromosomal instability (CI) in the progression of HPIN foci to invasive cancer and whether is that influenced by heterogeneity in the p53 expression between different HPIN foci is still unknown.

The objectives of this project are as follows. First, to study the p53 mutation pattern in HPIN foci that are intermingled with cancer and to compare them with different isolated HPIN foci situated in the same gland but away from any cancer foci. Second, to study the relation between p53 mutation and CI in precancerous and malignant prostate epithelium.

\section{MATERIAL AND METHODS}

\section{Patients}

Tissue samples were obtained from prostate carcinoma resected at Toronto General Hospital and Sunnybrook and Women's College Health Sciences Center, Toronto, Ontario, Canada. A total of 35 cases were selected based on the presence of HPIN foci intermingled with cancer and HPIN foci separated from cancer, with no other cancer foci in the serial blocks superior and inferior to that particular foci.

\section{Immunohistochemistry Staining}

Immunohistochemistry (IHC) was performed on archival formalin-fixed, paraffin-embedded sections $(5 \mu \mathrm{m})$ from prostatectomy specimens containing both prostate carcinoma and HPIN foci. The appropriate control was used. Monoclonal antibody to p53 (DO7 clone; Novocastra Laboratories Ltd., Newcastle, United Kingdom) was applied using avidin-biotin peroxidase complex (Elite kit; Vector Laboratories, Burlingame, CA). The positive control for p53 immunoreactivity consisted of formalin-fixed sections from an adenocarcinoma of breast and bladder transitional cell carcinoma. Negative internal controls were stromal cells. Immunoreactivity (IR) was categorized semiquantitatively from 0 to $4+(0=$ no IR, $1+=1-10 \%, 2+=$ $11-40 \%, 3+=41-70 \%, 4+=71-100 \%)$. Staining was defined as positive whenever any specific nuclear brown staining was detected. In the event of disagreement in quantification, the sample was rereviewed by both observers, and a consensus score was achieved.

\section{Interphase FISH Analysis}

Interphase FISH has been performed on $5-\mu \mathrm{m}$ unstained tissue sections of the same blocks used for the p53 study, using adjacent hematoxylin and eosin (H\&E) -stained sections as guidance. Directly labeled VYSIS CEP probes for chromosomes 7, 8, X, and $\mathrm{Y}$ were used. Paraffin pretreatment and FISH procedure were performed according to manufacturer instructions (Vysis, Inc., Downers Grove, IL). Dual-probe hybridization was performed. For each probe, 100 nuclei were counted by each observer. Chromosome $\mathrm{X}$ was used as an internal hybridization control for chromosome $\mathrm{Y}$ to determine whether any apparent loss of Y was caused by inadequate hybridization. The chromosome $\mathrm{X}$ signals were not enumerated.

\section{Criteria for Scoring and Evaluation of Numerical Chromosomal Anomalies}

In preliminary experiments, the hybridization efficiency of every probe has been tested on prostate tissues. Slides were evaluated according to the accepted criteria (31). Briefly, only sections with hybridization in at least $80 \%$ of cells were evaluated. For each probe, two independent investigators counted the number of FISH signals in 200 nonoverlapped intact (spherical) interphase nuclei from foci of HPIN. The number of signals per nucleus were scored as in terms of signal per nucleus: $0,1,2,3,4$, and more than 4 . Nuclei from stromal element were not enumerated. Normal and hyperplastic glandular epithelium present in the biopsies were counted as internal control. Because of truncation of the nuclei, artifact loss of signals was expected; however, we applied very conservative criteria to detect any significant true numeric changes. Our criteria to evaluate numeric chromosomal abnormality were as follows:

1) Chromosomal gains had been diagnosed when more than $8 \%$ of the nuclei exhibited more than two signals (or one for chromosome $\mathrm{Y}$ ).

2) Chromosomal losses had been diagnosed when more than $50 \%$ of the nuclei exhibited a reduction of signal number.

3) Tetraploidy had been suspected when the percentage of nuclei with three and four signals (or two for Y chromosome) was similar for both chromosomes 7 and 8 . These cutoff values were adopted from the available literature (32-36). In our study, as in others, no BPH specimens or normal prostate epithelium contained values that exceeded these criteria.

p53 Sequencing: Laser Capture Microdissection and Genomic DNA Extraction

Laser capture microdissection (LCM) was performed with a Pixcell II Laser Capture Microscope (Arcturus Engineering, Mountain View, CA) in the Ontario Cancer Institute. Tissue $(4-5-\mu \mathrm{m}$ thickness) were used, and foci of choice were dissected 
as described elsewhere $(37,38)$. DNA was extracted as previously described (39). DNA was analyzed for p53 mutation by the p53 sequencing method. DNA sequences of p53 (Exons 5-9) were amplified by PCR. Sequencing analysis was done using the p53 Mutation Detection GeneKit (Visible Genetics Inc., Toronto, Ontario, Canada). Each exon has been sequenced separately using 3 ' primer, and for those with any abnormality, the other, 5 ' direction was done to confirm the findings.

\section{Statistical Analysis}

The McNemar test was used to examine the differences between HPINA and HPINI in the same gland regarding p53 positivity and numeric chromosomal changes. The $z$ test was used to analyze the difference between p53+ and p53- HPIN regarding CI. The same test was used to examine the difference between p53 + and p53- Pca regarding CI.

\section{RESULTS}

We identified 35 prostatectomy specimens that have Pca with intermingled HPIN foci and at the same time have HPIN foci that are completely separated from the cancer foci and admixed with benign epithelium (Fig. 1). We performed p53 analyses using IHC (DO7) on representative sections of these specimens (total: 80 HPIN foci and 44 Pca foci). Table 1 summarizes the overall p53 and chromosomal anomalies in Pca, HPINI, HPINA, and benign prostate epithelium. Seven cases (20\%) stained positively for p53 in Pca foci (Fig. 2A-C). Immunoreactivity in those positive cases was categorized semiquantitatively as follows: 3 cases as $1+$, 2 cases as $2+$, and 2 cases as $3+$. There was a remarkable similarity between HPINI and Pca in the p53 immunoreactivity because six of those seven cases $(86 \%)$ also stained positively in the HPINI (Fig. 2A-D). None of those 7 cases showed immunoreactivity in the HPINA in the same glands (Fig. 2E). None of the p53-negative cancers showed positively in the HPIN foci. The normal, atrophic, and hyperplastic tissue situated in the same sections showed negative staining in all the cases (Fig. 2F). The Gleason grades for p53-positive cases were 7 (5 cases) and 8 ( 1 case) and 9 (1 case). The Gleason grades for p53-negative cases were 6 (11 cases), 7 (15 cases), 8 ( 1 case), and 9 ( 1 case). The volume of the tumor as evaluated by the percentage of the tumor in the gland for p53-positive cases was less than $10 \%$ in 4 cases and more than $10 \%$ in 3 cases. For p53-positive cases, the percentage of tumor was less than $10 \%$ in 17 cases and more than $10 \%$ in 11 cases. When this results were compared with pathological findings, there was no statistically sig- nificant difference between the p53-positive and p53-negative cases regarding Gleason grade, volume of the tumor, perineural invasion, seminal vesicle involvement, and lymph node metastasis. In five of seven p53+ cases, pathological examination showed extraprostatic extension, and that finding was seen in 10 patients out of 28 of the p53-negative cases $(P=.1)$. Focal cytoplasmic staining was seen in four cases (Patients 3, 5, 11 and 20) in the Pca and HPIN foci and was counted as negative. Focal (scattered cells) p53 basal staining was seen in about $30 \%$ of the cases in the hyperplastic foci but was found very rarely in HPIN foci. p53 sequencing analysis was performed for Exons 5-9 using a lasercaptured microdissected specimens from Pca, HPIN foci, and benign epithelium (Fig. 3) from 2 selected patients (one positive and one negative for p53 by IHC). In the patient with p53 immunoreactivity (Patient 34), sequencing analysis revealed that the tumor foci harbored point mutation TGT at Codon 273 instead of wild-type TGC in the highly conserved transcript region at Exon 8 substituting the encoded amino acid from arginine to cystine (Figure 4). The mutation has been confirmed using primers from both 3 ' and 5' direction. The other patient (Patient 9) with a negative p53 by IHC showed CI in cancer foci, so p53 sequencing analysis has been performed on normal foci, HPIN, and cancer from that patient to see whether cancer foci harbor termination mutation in p53 that might be missed by IHC. The analysis, however, revealed that all those foci harbor normal Exon 5-9 sequences, indicative of wild-type p53. The analysis of these two cases confirmed the IHC findings. IFISH analysis for chromosomes 7, 8, and $\mathrm{Y}$ was performed to assess $\mathrm{CI}$ in sections from the same blocks used for IHC analysis. Numeric chromosomal aberrations were found in $27 \%$ of HPINI and in $47 \%$ of Pca (Figs. 5-6). There were no statistically significant differences in the frequency of chromosomal anomalies between HPINI and Pca, and the overall frequencies of numeric chromosomal anomalies between them were similar. Numeric chromosomal aberrations were found in 5/7 and 4/6 of the p53+ Pca and p53+ HPIN, respectively. On the other hand, numeric chromosomal abnormality has been seen only in $2 / 8$ of p53- Pca and in none of the p53HPIN, including both HPIN that intermingled with cancer and those situated away. However, this finding was not found to be statistically significant. Gain of chromosome 8 was the most frequent change in both HPIN and Pca, followed by gain of chromosome 7. Chromosome $\mathrm{Y}$ aneusomy was seen in 2 cases of Pca (in Patient 6 as Y chromosome gain and in Patient 26 as Y chromosome loss), and in both cases the intermingled HPIN foci showed similar changes. No CI has been detected in the normal, hyperplastic, or atrophic epithelium. It 

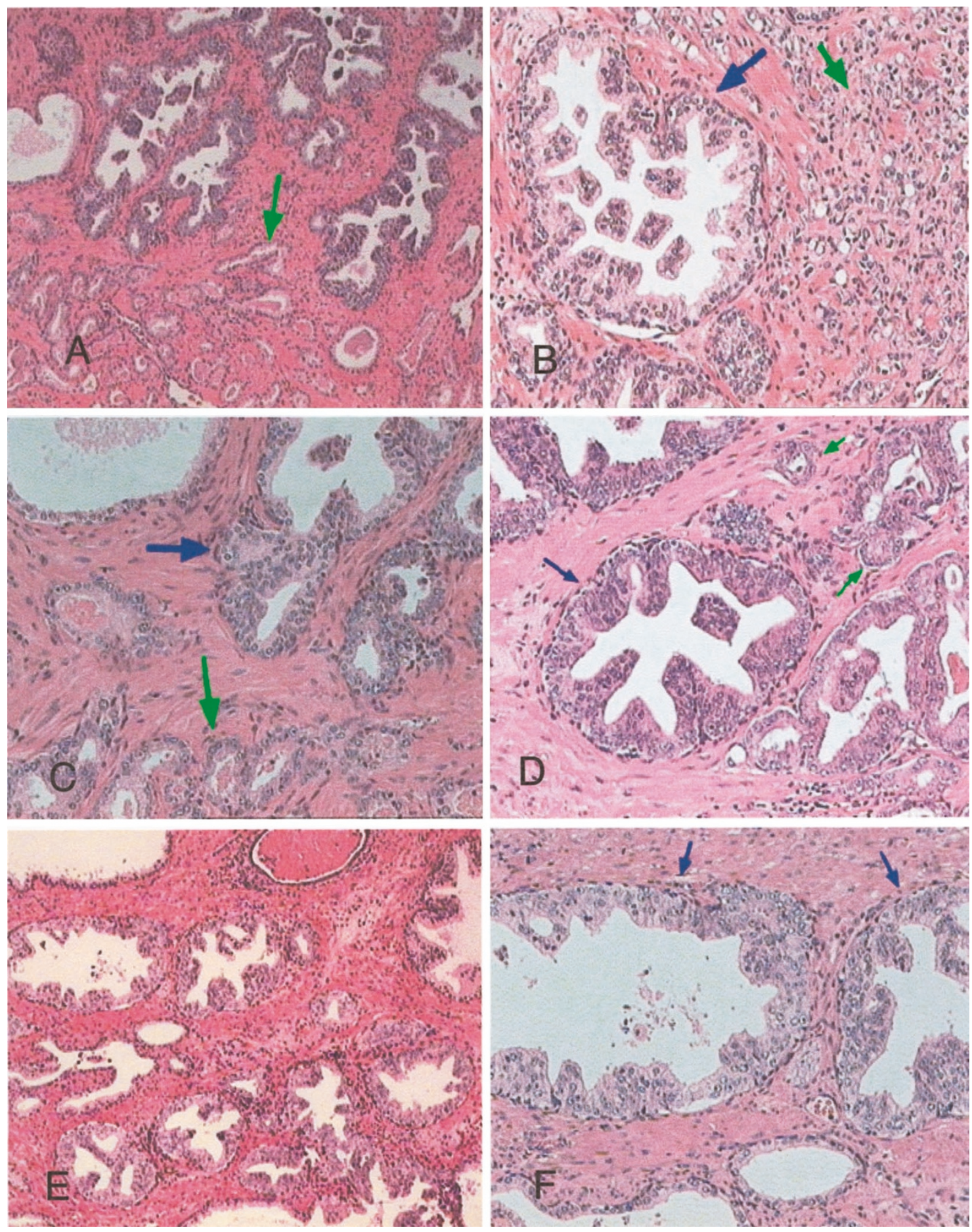

FIGURE 1. A-D, H\&E sections show foci of HPIN (blue arrow) intermingled with invasive cancer (green arrow). E, H\&E section of HPIN surrounded by benign epithelium. F, a higher power; HPIN foci (blue arrow) and benign epithelium in the lower part of the image.

was interesting to notice occasionally that early stromal invasion, the earliest morphologic indication of carcinomas, occurs at sites of acinar outpouching and basal cell disruption in acini with HPIN.

\section{DISCUSSION}

In agreement with results of other studies $(6,7$, 40-44) our results showed that p53 mutation oc- curs relatively infrequently in Pca (20\%) compared with the case of other human cancers like colon, esophagus, and lung cancer. However, the presence of HPIN foci with positive staining for p53 indicated that in a subset of Pca, the mutation could occur at an earlier stage of cancer pathogenesis. Our study showed heterogeneity of p53 positivity in the HPIN foci in the same gland, where foci of HPIN intermingled with p53-positive cancer foci tend to have 
TABLE 1. Summary of the p53 and Interphase FISH Results on Prostatectomy Specimens

\begin{tabular}{lccr}
\hline Group & Pca (\%) & HPINI (\%) & HPINA \\
\hline Total & 35 & 35 & 35 \\
P53+ (n:35) & $7(20)$ & $6(17.1)$ & 0 \\
CIN+ (n:15) & $7(47)$ & $4(27)$ & 0 \\
P53+/CIN+ & 5 & 4 & 0 \\
P53+/CIN- & 2 & 2 & 0 \\
P53-/CIN+ & 2 & 0 & 0 \\
P53-/CIN- & 6 & 9 & 0 \\
\hline
\end{tabular}
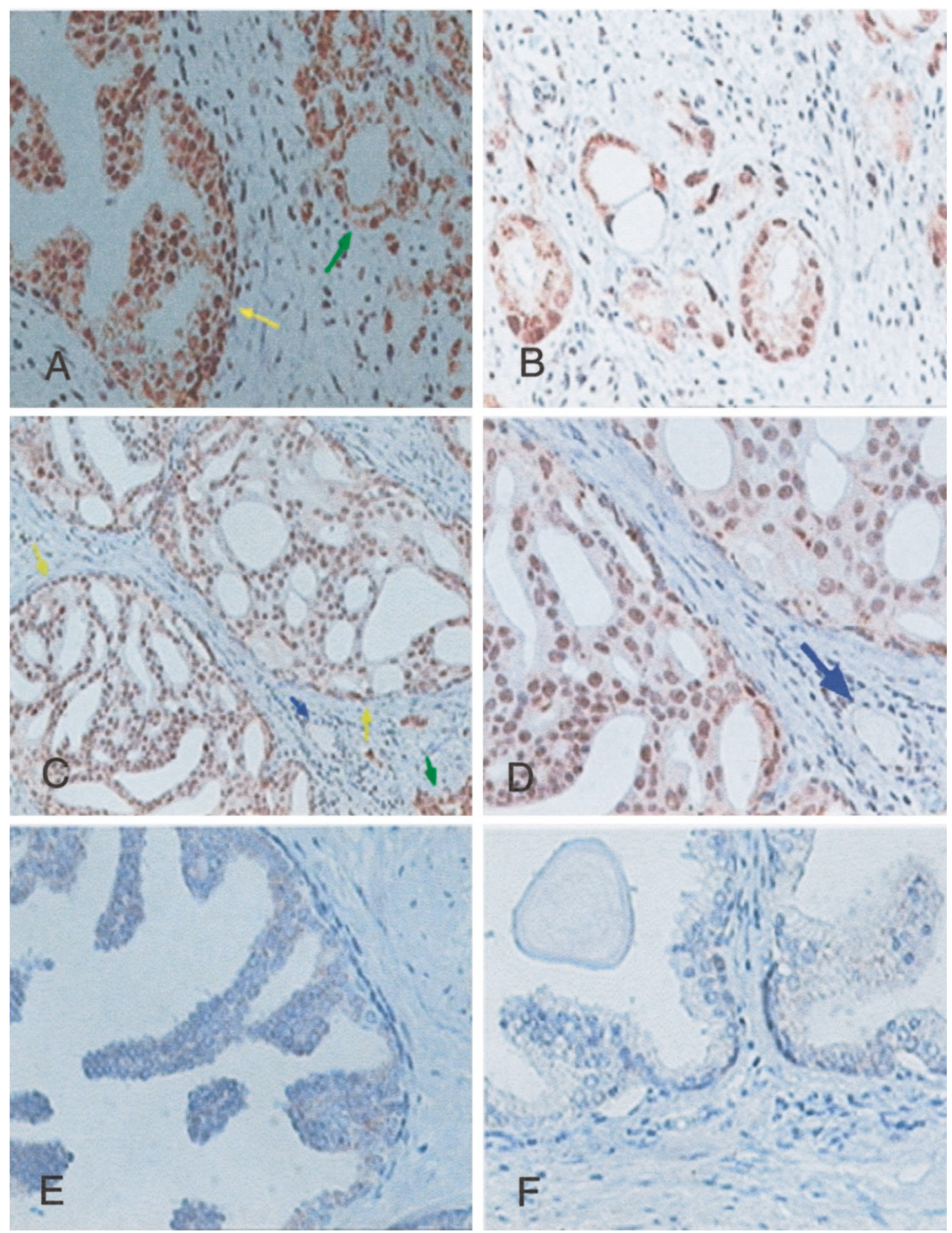

FIGURE 2. p53 IHC (DO7). A, positive nuclear staining in invasive cancer (green arrow) and in the adjacent HPIN (yellow arrow). B, positivity in cancer gland. C, another case with the same features. Blue arrow, vessels used as a negative control. D, high power. E, HPIN away from cancer with negative staining in the same Gland A. F, negative staining in a hyperplastic epithelium. 


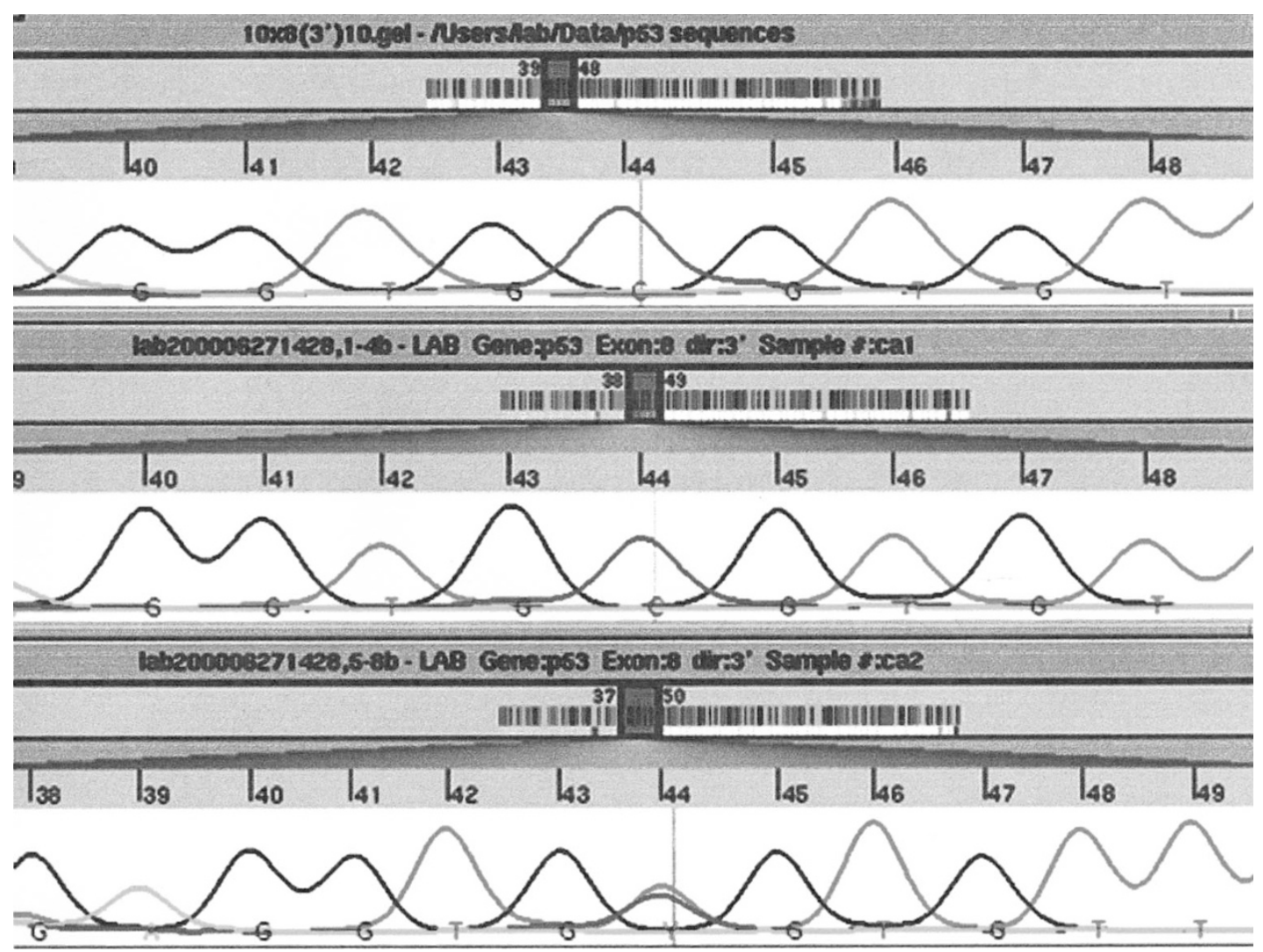

FIGURE 3. p53 sequencing analysis. Top picture, a normal sequence of Exon 8. Middle picture, a wild-type p53 (Patient 9). Bottom picture, mutated p53 with a change in Codon 273, changing the wild-type TGC to TGT and changing the amino acid from arginine to cystine. The corresponding IHC for p53 is on the left side.

higher incidence of p53 alteration than do isolated HPIN situated away from cancer and admixed with benign epithelium. Although $86 \%$ of HPINI in p53+ Pca showed p53 positivity, none of the HPINA were positive $(P<.05)$. That may explain some of the controversy in the literature regarding the incidence of p53 mutation in HPIN. Our study did not show positive nuclear staining in the adjacent normal, hyperplastic, or atrophic foci, including those tissues adjacent to or intermingled with cancer foci in any of the cases. In addition, CI was not observed in normal, hyperplastic, or atrophic epithelium. Taken together, these findings are not in keeping with the recently proposed idea that atrophy may give rise to carcinoma (45). More than $98 \%$ of all p53 mutations are located in Exons 5-9 (46, 47). We have performed p53 sequence analysis for Exons 5-9. Sequencing analysis has been performed using laser-captured microdissected specimens from Pca, HPIN foci, and benign epithelium. It has been done on a subset of cases (samples from 2 patients) to confirm the IHC findings. We have applied a laser capture microdissection technique that enables us to dissect very pure Pca and HPIN foci with no contamination. The discrepancies between IHC and PCR-SSCP that have been reported by some researchers in Pca could be caused by contamina- tion by normal tissue or foci without an apparent mutation because of the heterogeneity of Pca. Still, IHC does not detect all alteration that may affect p53 function, such as loss of heterozygosity at the p53 locus, nonsense or splice site mutations, or amplification of the MDM-2 gene, but all of these are very rare in prostate cancer. Generally, a good correlation between p53 alteration detected by IHC and molecular studies has been noted in prostate cancer (4, 7, 44, 48, 49). Hall et al. (44) found complete agreement between IHC and TP53 SSCP analysis. Wertz et al. (48) reported $85 \%$ overall agreement between the two methods, whereas the concordance was $76.7 \%$ by Salem et al. (7). In one of our cases, a point mutation has been seen at Codon 273 , changing the amino acid from arginine to cystine. p53 mutation at Codon 273 has been described in Pca $(10,50-52)$. G:C-to-A:T transitions were the most common point mutations $(64 \%)$ in prostate cancer (10). Six (55\%) of 11 G:C-to-A:T transitions occurred at $\mathrm{CpG}$ dinucleotides in five hot-spot codons $(175,245,248,273$, and 282$)$, and it was suggested that specific p53 mutations participate in the progression of human prostate cancer and may be predictive of metastasis (10).

This study, in addition to some other recent studies (both in vitro and in vivo), has demonstrated 

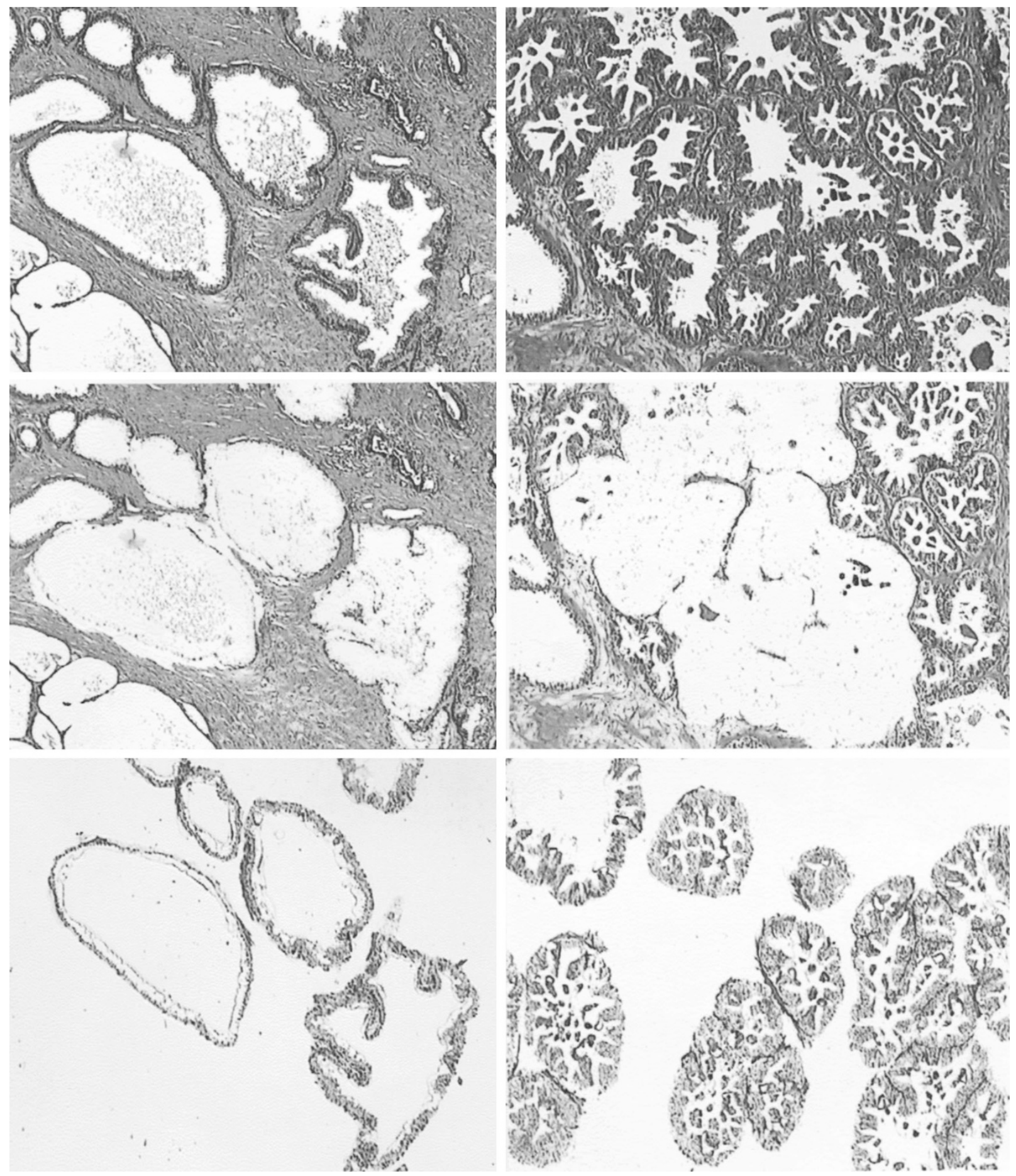

FIGURE 4. H\&E sections show an example of LCM. Dissection of benign epithelium (left side) and of HPIN (right side). Top pictures represent the tissue before dissection, middle pictures are after dissection, and bottom pictures are the cap tissues, which were used for p53, automated sequencing analysis.

correlation between loss or mutation of p53 and the presence of CI (53-63). More recently, centrosome hyperamplification was found to be the major mechanism responsible for $\mathrm{CI}$ in vitro and in vivo (58, 59, 64-66). Centrosome is the major microtubule-organizing center and is required for spindle bipolarity, spindle microtubule assembly, and balanced segregation of the chromosomes (67).
A very strong correlation has been found between p53 loss or mutation and centrosome hyperamplification $(27,55,59,67)$. Breast carcinoma and squamous cell carcinoma of the head and neck with either p53 deletion or mutation show centrosome hyperamplification $(58,64,65)$.

IFISH analysis for chromosomes 7, 8, and Y was performed to assess CI. We used these chromo- 


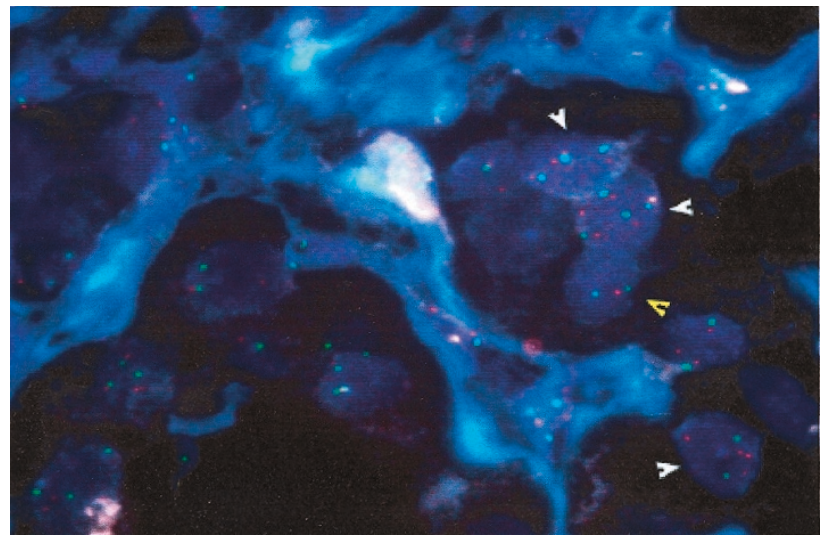

FIGURE 5. Interphase FISH on a focus of invasive prostate carcinoma using dual-centromere probes. Some cells (white arrowheads) show more than 2 green and more than 2 red signals, consistent with a gain of chromosomes 7 and 8 . The yellow arrowhead shows cells with 2 green and 1 red signals.

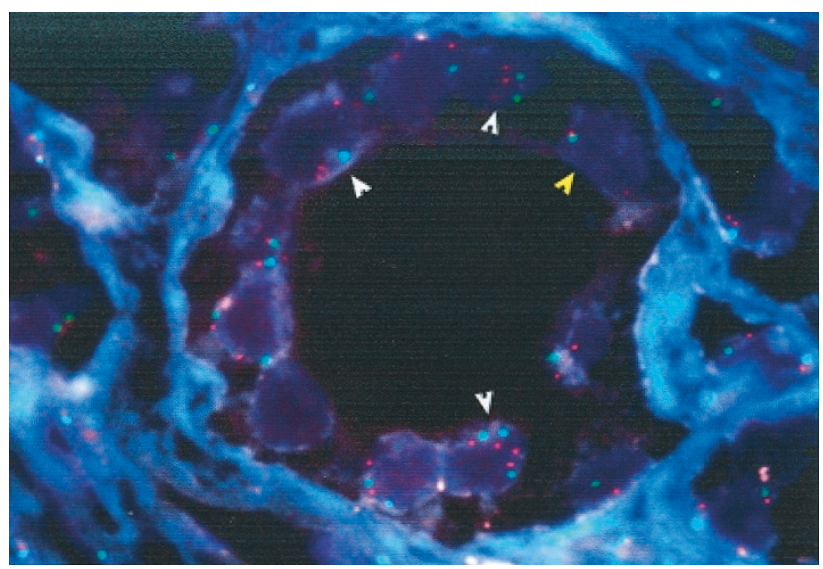

FIGURE 6. Interphase FISH on a focus of invasive prostate carcinoma using dual probes. Some cells (white arrowheads) show 3 green signals and 4 red signals, consist with a gain of chromosomes 7 and 8. Other cells (yellow arrowhead) show only one red and one green signal.

somes to assess CI because they are the most frequently affected chromosomes in prostate cancer pathogenesis. Although CI represents generalized changes in the cellular chromosomes, it is selective for certain chromosomes in carcinogenesis of different organs. Our finding revealed numeric chromosomal aberrations in 5/7 and 2/8 of p53-positive and p53-negative Pca, respectively $(P=.1)$. However, the presence of any numeric chromosomal abnormality has been seen in $4 / 6$ and $0 / 9$ of p53positive and p53-negative HPIN $(P<.02)$. Generally, none of the p53- intermingled and away HPIN foci showed any chromosomal abnormality. So generally, HPINI tend to have more CIN than those situated away (4/15 versus $0 / 15)$, with statistically significant difference $(P<.05)$. No $C I$ has been detected in the normal, hyperplastic, or atrophic epithelium and those areas showed no p53 alteration either. This suggest that those HPINI foci may represent the source of the adjacent invasive component, whereas the other isolated HPINA foci that admixed with benign epithelium may still be in the early stages of the carcinogenesis pathway and probably require more $\mathrm{CI}$ to progress to invasive cancer. This also suggested that p53 mutation may play a role in the progression of HPIN to invasive cancer, and this could happen through induction of CI.

We applied IFISH on sections from the same blocks that have been used for p53 IHC and that enabled us to compare the findings of the two assays in the same foci of tissue. IFISH has higher sensitivity than other methods used for this purpose, such as CGH, which detects copy number changes if they are present in more than $50 \%$ of the cell population (21). IFISH can identify CI in small subpopulations of interphase cells (68), allowing the detection of infrequent, possibly random changes before they lead to clonal expansion (20). Using IFISH on pretreatment and post antiandrogen therapy prostate cancer specimens, Karashima et al. (69) found a remarkable reduction in the number of cells with extra copies of chromosomes 7 and 8.

Our IFISH results showed that gain of chromosome 8 is the most frequent finding in both HPIN and Pca. c-Myc gene is located in the $8 \mathrm{q}$ arm, and gain of chromosome 8 indicated an extra copy of that important oncogene. The role of c-Myc in the mechanism of CI has been recently described. Extra copies of the c-Myc gene were identified in 52 and $44 \%$ of the high-grade PIN and carcinoma foci, respectively (70), and by Mark et al. (71) in $31 \%$ of Pca. In some cancers displaying CI, the loss of the checkpoint was associated with the mutational inactivation of a human homologue of the yeast BUB1 gene. BUB1 controls mitotic checkpoints and chromosome segregation in yeast (72). Disruption of the mitotic spindle checkpoint is one of the underlying mechanisms leading to aneuploidy and alterations of hsMAD2 and hBUB1. This mechanism, assumed to take part in the spindle checkpoint in human cells, has been found to be associated with $\mathrm{CI}$ in some tumor cell lines (8). However, there is no study on these genes in prostate tumors. Other possible mechanisms may be involved in the causation of CI, such as shortened telomeres, hypomethylation, activation of certain genes or inactivation of tumor suppressor genes.

\section{CONCLUSION}

We demonstrated that p53 mutation is an early change in at least a subset of Pca. HPINI foci tend to have higher overall p53 immunoreactivity and CI than HPINA. The presence of p53 mutation in HPIN 
was associated with the presence of $\mathrm{CI}$ as determined by IFISH. Also, our study provided additional evidence in support of the concept that HPIN is the earliest precursor of cancer. Furthermore our studies identify genomic similarities in HPINI and Pca, implying that carcinoma may arise from progression of certain HPIN foci that most likely harbor p53 mutation and/or elevated levels of CI.

Acknowledgment: We thank our lab members (Khladoun Alromaih, Jane Bayani, Ben Beheshti, Jana Karaskova, Elena Kolomietz, Paula Marrano, Paul Park, Bisera Vukovic, and Zong Mei Zhang) for their contributions to this work.

\section{REFERENCES}

1. Tamboli P, Amin MB, Xu HJ, Linden MD. Immunohistochemical expression of retinoblastoma and p53 tumor suppressor genes in prostatic intraepithelial neoplasia: comparison with prostatic adenocarcinoma and benign prostate. Mod Pathol 1998;11:247-52.

2. Gumerlock PH, Chi SG, Shi XB, Voeller HJ, Jacobson JW, Gelmann EP, et al. p53 abnormalities in primary prostate cancer: single-strand conformation polymorphism analysis of complementary DNA in comparison with genomic DNA. The Cooperative Prostate Network. J Natl Cancer Inst 1997; 89:66-71.

3. Heidenberg HB, Bauer JJ, McLeod DG, Moul JW, Srivastava S. The role of the p53 tumor suppressor gene in prostate cancer: a possible biomarker? Urology 1996;48:971-9.

4. Navone NM, Troncoso P, Pisters LL, Goodrow TL, Palmer JL, Nichols WW, et al. p53 protein accumulation and gene mutation in the progression of human prostate carcinoma. J Natl Cancer Inst 1993;85:1657-69.

5. Thomas DJ, Robinson M, King P, Hasan T, Charlton R, Martin J, et al. p53 expression and clinical outcome in prostate cancer. Br J Urol 1993;72:778-81.

6. Yasunaga Y, Shin M, Fujita MQ, Nonomura N, Miki T, Okuyama A, et al. Different patterns of p53 mutations in prostatic intraepithelial neoplasia and concurrent carcinoma: analysis of microdissected specimens. Lab Invest 1998; 78:1275-9.

7. Salem CE, Tomasic NA, Elmajian DA, Esrig D, Nichols PW, Taylor CR, et al. p53 protein and gene alterations in pathological stage C prostate carcinoma. J Urol 1997;158:510-4.

8. Imai $\mathrm{Y}$, Shiratori $\mathrm{Y}$, Kato N, Inoue T, Omata M. Mutational inactivation of mitotic checkpoint genes, hsMAD2 and hBUB1, is rare in sporadic digestive tract cancers. Jpn J Cancer Res 1999;90:837-40.

9. Massenkeil G, Oberhuber H, Hailemariam S, Sulser T, Diener PA, Bannwart F, et al. P53 mutations and loss of heterozygosity on chromosomes $8 \mathrm{p}, 16 \mathrm{q}, 17 \mathrm{p}$, and $18 \mathrm{q}$ are confined to advanced prostate cancer. Anticancer Res 1994;14:278590.

10. Navone NM, Labate ME, Troncoso P, Pisters LL, Conti CJ, von Eschenbach AC, et al. p53 mutations in prostate cancer bone metastases suggest that selected p53 mutants in the primary site define foci with metastatic potential. J Urol 1999;161:304-8.

11. Stricker HJ, Jay JK, Linden MD, Tamboli P, Amin MB. Determining prognosis of clinically localized prostate cancer by immunohistochemical detection of mutant p53. Urology 1996;47:366-9.
12. Cheng L, Leibovich BC, Bergstralh EJ, Scherer BG, Pacelli A, Ramnani DM, et al. p53 alteration in regional lymph node metastases from prostate carcinoma: a marker for progression? Cancer 1999;85:2455-9.

13. Meyers FJ, Gumerlock PH, Chi SG, Borchers H, Deitch AD, deVere White RW. Very frequent p53 mutations in metastatic prostate carcinoma and in matched primary tumors. Cancer 1998;83:2534-9.

14. Johnson MI, Robinson MC, Marsh C, Robson CN, Neal DE, Hamdy FC. Expression of Bcl-2, Bax, and p53 in high-grade prostatic intraepithelial neoplasia and localized prostate cancer: relationship with apoptosis and proliferation. Prostate 1998;37:223-9.

15. Humphrey PA, Swanson PE. Immunoreactive p53 protein in high-grade prostatic intraepithelial neoplasia. Pathol Res Pract 1995;191:881-7.

16. Mirchandani D, Zheng J, Miller GJ, Ghosh AK, Shibata DK, Cote RJ, et al. Heterogeneity in intratumor distribution of p53 mutations in human prostate cancer. Am J Pathol 1995; 147:92-101.

17. MacGrogan D, Bookstein R. Tumour suppressor genes in prostate cancer. Semin Cancer Biol 1997;8:11-9.

18. Veltman JA, Hopman AH, van der Vlies SA, Bot FJ, Ramaekers FC, Manni JJ. Double-target fluorescence in situ hybridization distinguishes multiple genetically aberrant clones in head and neck squamous cell carcinoma. Cytometry 1998; 34(3):113-20.

19. Veltman JA, Bot FJ, Huynen FC, Ramaekers FC, Manni JJ, Hopman AH. Chromosome instability as an indicator of malignant progression in laryngeal mucosa. J Clin Oncol 2000;18:1644-51.

20. Rabinovitch PS, Dziadon S, Brentnall TA, Emond MJ, Crispin DA, Haggitt RC, et al. Pancolonic chromosomal instability precedes dysplasia and cancer in ulcerative colitis. Cancer Res 1999;59:5148-53.

21. Ried T, Heselmeyer-Haddad K, Blegen H, Schrock E, Auer G. Genomic changes defining the genesis, progression, and malignancy potential in solid human tumors: a phenotype/ genotype correlation. Genes Chromosomes Cancer 1999;25: 195-204.

22. Burt EC, James LA, Greaves MJ, Birch JM, Boyle JM, Varley JM. Genomic alterations associated with loss of heterozygosity for TP53 in Li-Fraumeni syndrome fibroblasts. Br J Cancer 2000;83:467-72.

23. Hawkins NJ, Gorman P, Tomlinson IP, Bullpitt P, Ward RL. Colorectal carcinomas arising in the hyperplastic polyposis syndrome progress through the chromosomal instability pathway. Am J Pathol 2000;157:385-92.

24. Shackney SE, Shankey TV. Common patterns of genetic evolution in human solid tumors. Cytometry 1997;29(1):1-27.

25. Williams AC, Miller JC, Collard T, Browne SJ, Newbold RF, Paraskeva C. The effect of different TP53 mutations on the chromosomal stability of a human colonic adenoma derived cell line with endogenous wild type TP53 activity, before and after DNA damage. Genes Chromosomes Cancer 1997;20:44-52.

26. Yasui W, Yokozaki H, Fujimoto J, Naka K, Kuniyasu H, Tahara E. Genetic and epigenetic alterations in multistep carcinogenesis of the stomach. J Gastroenterol 2000;35(12 Suppl):111-5.

27. Tarapore P, Fukasawa K. p53 mutation and mitotic infidelity. Cancer Invest 2000;18:148-55.

28. Kirchhoff M, Rose H, Petersen BL, Maahr J, Gerdes T, Lundsteen $\mathrm{C}$, et al. Comparative genomic hybridization reveals a recurrent pattern of chromosomal aberrations in severe dysplasia/carcinoma in situ of the cervix and in advanced-stage cervical carcinoma. Genes Chromosomes Cancer 1999;24:144-50.

29. Mian C, Bancher D, Kohlberger P, Kainz C, Haitel A, Czerwenka $\mathrm{K}$, et al. Fluorescence in situ hybridization in cervical smears: detection of numerical aberrations of chromosomes 
7, 3, and X and relationship to HPV infection. Gynecol Oncol 1999;75:41-6.

30. Aubele M, Zitzelsberger H, Schenck U, Walch A, Hofler H, Werner M. Distinct cytogenetic alterations in squamous intraepithelial lesions of the cervix revealed by laser-assisted microdissection and comparative genomic hybridization. Cancer 1998;84:375-9.

31. Henke RP, Kruger E, Ayhan N, Hubner D, Hammerer P. Frequency and distribution of numerical chromosomal aberrations in prostatic cancer. Hum Pathol 1994;25:476-84.

32. Qian J, Bostwick DG, Takahashi S, Borell TJ, Brown JA, Lieber $\mathrm{MM}$, et al. Comparison of fluorescence in situ hybridization analysis of isolated nuclei and routine histological sections from paraffin-embedded prostatic adenocarcinoma specimens. Am J Pathol 1996;149:1193-9.

33. Erbersdobler A, Bardenhagen P, Henke RP. Numerical chromosomal anomalies in latent adenocarcinomas of the prostate. Prostate 1999;38:92-9.

34. Erbersdobler A, Gurses N, Henke RP. Numerical chromosomal changes in high-grade prostatic intraepithelial neoplasia (PIN) and concomitant invasive carcinoma. Pathol Res Pract 1996;192:418-27.

35. Aubele M, Zitzelsberger H, Szucs S, Werner M, Braselmann $\mathrm{H}$, Hutzler $\mathrm{P}$, et al. Comparative FISH analysis of numerical chromosome 7 abnormalities in 5- micron and 15-micron paraffin-embedded tissue sections from prostatic carcinoma. Histochem Cell Biol 1997;107:121-6.

36. Qian J, Bostwick DG, Takahashi S, Borell TJ, Herath JF, Lieber MM, et al. Chromosomal anomalies in prostatic intraepithelial neoplasia and carcinoma detected by fluorescence in situ hybridization. Cancer Res 1995;55:5408-14.

37. Ornstein DK, Englert C, Gillespie JW, Paweletz CP, Linehan WM, Emmert-Buck MR, et al. Characterization of intracellular prostate-specific antigen from laser capture microdissected benign and malignant prostatic epithelium. Clin Cancer Res 2000;6:353-6.

38. Emmert-Buck MR, Bonner RF, Smith PD, Chuaqui RF, Zhuang Z, Goldstein SR, et al. Laser capture microdissection. Science 1996;274:998-1001.

39. Ehrig T, Abdulkadir SA, Dintzis SM, Milbrandt J, Watson MA. Quantitative amplification of genomic DNA from histological tissue sections after staining with nuclear dyes and laser capture microdissection. J Mol Diagn 2001;3:22-5.

40. Yang G, Stapleton AM, Wheeler TM, Truong LD, Timme TL, Scardino PT, et al. Clustered p53 immunostaining: a novel pattern associated with prostate cancer progression. Clin Cancer Res 1996;2:399-401.

41. Ruijter E, van de Kaa C, Aalders T, Ruiter D, Miller G, Debruyne F, et al. Heterogeneous expression of E-cadherin and p53 in prostate cancer: clinical implications. BIOMED-II Markers for Prostate Cancer Study Group. Mod Pathol 1998; 11:276-81.

42. Schlechte HH, Schnorr D, Loning T, Rudolph BD, Pohrt UM, Loening SA. Mutation of the tumor suppressor gene p53 in human prostate and bladder cancers-investigation by temperature gradient gel electrophoresis (TGGE). J Urol 1997; 157:1049-53

43. Stattin P, Bergh A, Karlberg L, Nordgren H, Damber JE. p53 immunoreactivity as prognostic marker for cancer-specific survival in prostate cancer. Eur Urol 1996;30:65-72.

44. Hall MC, Navone NM, Troncoso P, Pollack A, Zagars GK, von Eschenbach AC, et al. Frequency and characterization of p53 mutations in clinically localized prostate cancer. Urology 1995;45:470-5.

45. De Marzo AM, Marchi VL, Epstein JI, Nelson WG. Proliferative inflammatory atrophy of the prostate: implications for prostatic carcinogenesis. Am J Pathol 1999;155:1985-92.

46. Levine AJ, Momand J, Finlay CA. The p53 tumour suppressor gene. Nature 1991;351:453-6.
47. Hollstein M, Sidransky D, Vogelstein B, Harris CC. p53 mutations in human cancers. Science 1991;253:49-53.

48. Wertz IE, Deitch AD, Gumerlock PH, Gandour-Edwards R, Chi SG, de Vere White RW. Correlation of genetic and immunodetection of TP53 mutations in malignant and benign prostate tissues. Hum Pathol 1996;27:573-80.

49. Jacquemier J, Moles JP, Penault-Llorca F, Adelaide J, Torrente $\mathrm{M}$, Viens $\mathrm{P}$, et al. p53 immunohistochemical analysis in breast cancer with four monoclonal antibodies: comparison of staining and PCR-SSCP results. Br J Cancer 1994;69:846-52.

50. Carroll AG, Voeller HJ, Sugars L, Gelmann EP. p53 oncogene mutations in three human prostate cancer cell lines. Prostate 1993;23:123-34.

51. Dinjens WN, van der Weiden MM, Schroeder FH, Bosman FT, Trapman J. Frequency and characterization of p53 mutations in primary and metastatic human prostate cancer. Int J Cancer 1994;56:630-3.

52. Kunimi K, Amano T, Uchibayashi T. Point mutation of the p53 gene is an infrequent event in untreated prostate cancer. Cancer Detect Prev 1996;20:218-22.

53. Yin XY, Grove L, Datta NS, Long MW, Prochownik EV. C-myc overexpression and p53 loss cooperate to promote genomic instability. Oncogene 1999;18:1177-84.

54. Kanekawa A, Tsuji T, Oga A, Sasaki K, Shinozaki F. Chromosome 17 abnormalities in squamous cell carcinoma of the oral cavity, and its relationship with p53 and Bcl-2 expression. Anticancer Res 1999;19:81-6.

55. Eshleman JR, Casey G, Kochera ME, Sedwick WD, Swinler SE, Veigl ML, et al. Chromosome number and structure both are markedly stable in RER colorectal cancers and are not destabilized by mutation of p53. Oncogene 1998;17:719-25.

56. Kohno H, Hiroshima K, Toyozaki T, Fujisawa T, Ohwada H. p53 mutation and allelic loss of chromosome 3p, 9p of preneoplastic lesions in patients with nonsmall cell lung carcinoma. Cancer 1999;85:341-7.

57. Albertoni M, Daub DM, Arden KC, Viars CS, Powell C, Van Meir EG. Genetic instability leads to loss of both p53 alleles in a human glioblastoma. Oncogene 1998;16:321-6.

58. Carroll PE, Okuda M, Horn HF, Biddinger P, Stambrook PJ, Gleich LL, et al. Centrosome hyperamplification in human cancer: chromosome instability induced by p53 mutation and/or Mdm2 overexpression. Oncogene 1999;18:1935-44.

59. Fukasawa K, Wiener F, Vande Woude GF, Mai S. Genomic instability and apoptosis are frequent in p53 deficient young mice. Oncogene 1997;15:1295-302.

60. Agapova LS, Ilyinskaya GV, Turovets NA, Ivanov AV, Chumakov PM, Kopnin BP. Chromosome changes caused by alterations of p53 expression. Mutat Res 1996;354:129-38.

61. Smith ML, Fornace AJ Jr. Genomic instability and the role of p53 mutations in cancer cells. Curr Opin Oncol 1995;7:69-75.

62. Donehower LA, Godley LA, Aldaz CM, Pyle R, Shi YP, Pinkel $\mathrm{D}$, et al. Deficiency of $\mathrm{p} 53$ accelerates mammary tumorigenesis in Wnt-1 transgenic mice and promotes chromosomal instability. Genes Dev 1995;9:882-95.

63. Bouffler SD, Kemp CJ, Balmain A, Cox R. Spontaneous and ionizing radiation-induced chromosomal abnormalities in p53-deficient mice. Cancer Res 1995;55:3883-9.

64. Lingle WL, Lutz WH, Ingle JN, Maihle NJ, Salisbury JL. Centrosome hypertrophy in human breast tumors: implications for genomic stability and cell polarity. Proc Natl Acad Sci U S A 1998;95:2950-5.

65. Pihan GA, Purohit A, Wallace J, Knecht H, Woda B, Quesenberry $\mathrm{P}$, et al. Centrosome defects and genetic instability in malignant tumors. Cancer Res 1998;58:3974-85.

66. Ghadimi BM, Sackett DL, Difilippantonio MJ, Schrock E, Neumann T, Jauho A, et al. Centrosome amplification and instability occurs exclusively in aneuploid, but not in diploid colorectal cancer cell lines, and correlates with numerical 
chromosomal aberrations. Genes Chromosomes Cancer 2000;27:183-90.

67. Fukasawa K, Choi T, Kuriyama R, Rulong S, Vande Woude GF. Abnormal centrosome amplification in the absence of p53. Science 1996;271:1744-7.

68. Lengauer C, Kinzler KW, Vogelstein B. Genetic instability in colorectal cancers. Nature 1997;386:623-7.

69. Karashima T, Taguchi T, Yoshikawa C, Kamada M, Kasahara K, Yuri $\mathrm{K}$, et al. Numerical chromosomal changes in metastatic prostate cancer following anti-androgen therapy: fluorescence in situ hybridization analysis of 5 Japanese cases. Cancer Genet Cytogenet 2000;120:148-54.
70. Qian J, Jenkins RB, Bostwick DG. Detection of chromosomal anomalies and c-myc gene amplification in the cribriform pattern of prostatic intraepithelial neoplasia and carcinoma by fluorescence in situ hybridization. Mod Pathol 1997;10: 1113-9.

71. Mark HF, Samy M, Santoro K, Mark S, Feldman D. Fluorescent in situ hybridization study of c-myc oncogene copy number in prostate cancer. Exp Mol Pathol 2000;68: 65-9.

72. Cahill DP, Lengauer C, Yu J, Riggins GJ, Willson JK, Markowitz SD, et al. Mutations of mitotic checkpoint genes in human cancers. Nature 1998;392:300-3. 